\title{
Genetic Variation in Acid Ceramidase Predicts Non-completion of an Exercise Intervention
}

\begin{abstract}
Lauren S. Lewis ${ }^{1}$, Kim M. Huffman ${ }^{2,3}$, Ira J. Smith ${ }^{4}$, Mark P. Donahue ${ }^{4}$, Cris A. Slentz ${ }^{2}$, Joseph A. Houmard ${ }^{5}$, Monica J. Hubal ${ }^{6}$, Eric P. Hoffman ${ }^{6}$, Elizabeth R. Hauser ${ }^{2,7}$, llene C. Siegler ${ }^{8}$ and William E. Kraus ${ }^{2,4 *}$

${ }^{1}$ Department of Obstetrics and Gynecology, Duke University School of Medicine, Durham, NC, United States, ${ }^{2}$ Duke Molecular Physiology Institute, Duke University School of Medicine, Durham, NC, United States, ${ }^{3}$ Division of Rheumatology and Immunology, Department of Medicine, Duke University School of Medicine, Durham, NC, United States, ${ }^{4}$ Division of Cardiology, Department of Medicine, Duke University School of Medicine, Durham, NC, United States, ${ }^{5}$ Human Performance Laboratory, East Carolina University, Greenville, NC, United States, ${ }^{6}$ Children's Genetic Medical Research Center, Children's National Medical Center, Washington, DC, United States, ${ }^{7}$ Cooperative Studies Program-Epidemiology Center Durham, Veterans Administration Medical Center, Durham, NC, United States, ${ }^{8}$ Division of Behavioral Medicine, Department of Psychiatry, Duke University School of Medicine, Durham, NC, United States
\end{abstract}

Genetic variation is associated with a number of lifestyle behaviours; it may be associated with adherence and individual responses to exercise training. We tested single nucleotide polymorphisms (SNPs) in the acid ceramidase gene (ASAH1) for association with subject adherence and physiologic benefit with exercise training in two well-characterised randomised, controlled 8-month exercise interventions: STRRIDE I $(n=239)$ and STRRIDE II $(n=246)$. Three ASAH1 non-coding SNPs in a linkage disequilibrium block were associated with non-completion: rs2898458(G/T), rs7508(A/G), and rs3810(A/G) were associated with non-completion in both additive $(\mathrm{OR}=1.8,1.8,2.0 ; P<0.05$ all) and dominant $(\mathrm{OR}=2.5,2.6,3.5 ; P<0.05$ all) models; with less skeletal muscle ASAH expression $(p<0.01)$ in a subset $(N=60)$; and poorer training response in cardiorespiratory fitness (peak $\mathrm{VO}_{2}$ change rs3810 $r^{2}=0.29, P=0.04$; rs2898458 $r^{2}=0.29, P=0.08 ; r s 7508 r^{2}=0.28, p=0.09$ ); and similar in direction and magnitude in both independent exploratory and replication studies. Adherence to exercise may be partly biologically and genetically moderated through metabolic regulatory pathways participating in skeletal muscle adaptation to exercise training.

Keywords: STRRIDE, metabolism, ceramide, exercise adherence, behavioural lifestyle interventions

\section{INTRODUCTION}

Many of the health benefits of exercise are mediated by metabolic adaptations in skeletal muscle, and increases in cardiorespiratory fitness. As detailed in the Physical Activity Guidelines Advisory Committee Report the benefits of exercise are substantial (DHHS, 2008) however, perhaps the major clinical issue confronting the use of exercise training as a therapeutic option is how to get individuals to initiate an exercise training program and-following that-how to maintain it. Common wisdom holds that adherence issues are primarily related to neurobehavioural and social issues, and that barriers can be identified and addressed using behavioural approaches. Although most attention regarding adherence to lifestyle interventions has traditionally focused on 
psychosocial behavioural factors, it is conceivable that there also exist biological and genetic factors determining whether individuals maintain an exercise program once initiated.

An additional issue complicating the use of exercise training as a therapeutic option is the variability in training responses. Conventional wisdom holds that more exercise is better, and that exercise is beneficial for everyone for a myriad of health benefits. Rather, even when controlling for adherence, there is a range of health responses to any given exercise program for any given health parameter (e.g., fitness, blood lipids, insulin sensitivity, blood pressure control, even weight change). In sum, not all individuals respond in a similaror even in a favourable manner-to an exercise program (Bouchard et al., 2012). Such variation in biological response to a given exercise exposure is heritable; with heritability estimates ranging from 29 to $70 \%$. This suggests a large fraction of the exercise response is moderated by genetic factors. These observations raised the prospect that genetic classifiers might be assembled to predict the variability in responses to exercise.

Genes of metabolic pathways active in skeletal muscle are associated with exercise performance; (Bouchard et al., 2011) and many of the physiologic effects of exercise training result from the adaption of skeletal muscle mitochondria (Duscha et al., 2005; Huffman et al., 2014). Thus, genes involved in skeletal muscle mitochondrial function serve as important candidate genes in studying the heritability of compliance with and response to exercise training. One antagonist of mitochondrial function-ceramide-is a central compound in sphingolipid metabolism. Intracellular ceramide content is increased in obese individuals; and the accumulation of ceramide in nonadipocytes is implicated in many obesity-associated diseases: lipotoxic heart disease, atherosclerosis, and type 2 diabetes (Zhou et al., 2000; Birbes et al., 2002; Hannun and Obeid, 2002; Summers, 2006). Ceramide content is reduced with short exercise bouts and exercise training, although exercise effects on ceramide pathway components are complex and incompletely understood (Dobrzyn and Gorski, 2002a,b; Helge et al., 2004; Bruce et al., 2006; Baranowski et al., 2008; BłachnioZabielska et al., 2008, 2011; Bergman et al., 2016). Ceramide impinges on mitochondrial adaptations to exercise by directly inhibiting the respiratory chain, activating apoptotic pathways converging on mitochondria, and promoting programmed cell death and inflammation (Birbes et al., 2002; Hannun and Obeid, 2002; Summers, 2006; Mao and Obeid, 2008); these bioactive effects can be regulated via conversion of ceramide to alternate metabolites, including degradation by acid ceramidase (Mao and Obeid, 2008). Despite the regulatory roles of acid ceramidase on ceramide effects impacting exercise, to the best of our knowledge no one has investigated acid ceramidase genetic variants in exercise responses. We hypothesised that acid ceramidase genetic variants might impact ceramide metabolism and other downstream metabolic responses to exercise; exercise capacity; and thereby influence exercise adherence. Here, using two well-described randomised controlled exercise trials, we capitalised on a $35 \%$ non-completion rate to investigate the association of single nucleotide polymorphisms (SNPs) in the acid ceramidase (EC 3.5.1.23) gene (ASAH1) with the ability to predict completion of and response to exercise training.

\section{METHODS}

\section{Subjects}

Two independent exercise trial samples were available: STRRIDE I (NCT00200993) and STRRIDE II (NCT00275145). A complete description of the STRRIDE I design is published elsewhere (Kraus et al., 2001). In summary, subjects were: 40-65 years of age; sedentary (exercised less than once weekly); overweight or obese (BMI 25 to $35 \mathrm{~kg} / \mathrm{m} 2$ ); had fasting hyperinsulinemia $(>10 \mathrm{IU} / \mathrm{mL}$ ) with mild to moderate lipid abnormalities (LDL cholesterol between 130 and $190 \mathrm{mg} / \mathrm{dL}$ or HDL cholesterol $<45 \mathrm{mg} / \mathrm{dL}$ for women or $40 \mathrm{mg} / \mathrm{dL}$ for men). The study design was similar. STRRIDE II, subjects were: 18-70 years of age; similarly, overweight or obese; sedentary; and with mild lipid abnormalities (Table 1). All subjects provided verbal and written informed consent as approved by the of Duke University Investigational Review Board and ECU Investigational Review Board. Subjects meeting inclusion criteria were randomised to one of four exercise groups in each study. Those subjects with DNA available for genetics studies are studied in this report.

\section{Exercise Training}

In STRRIDE I, subjects were randomly assigned to one of four groups: (1) non-exercising control; (2) low volume/moderate intensity aerobic exercise, defined as a caloric equivalent of 12 miles/week at $40-55 \%$ peak oxygen consumption (peak $\mathrm{VO}_{2}$ ); (3) low volume/vigorous intensity, defined as the caloric equivalent of 12 miles/week at $65-80 \%$ peak $\mathrm{VO}_{2}$; and (4) high volume/vigorous intensity exercise, defined as the caloric equivalent of 20 miles/week at $65-85 \%$ peak $\mathrm{VO}_{2}$. Caloric equivalents were determined by the approximate energy expenditure during walking or jogging for a $90 \mathrm{~kg}$ person; however, actual exercise modalities included cycle ergometers, treadmills, and elliptical trainers. The subjects underwent a 2month ramp period in which exercise intensity and duration were gradually increased until the appropriate regimen was reached; this was followed by an additional 6 months of exercise training.

In STRRIDE II, subjects underwent a 3-month control runin period followed by an 8-month exercise intervention in one of four exercise groups: (1) low volume/vigorous intensity group, identical to the low volume/vigorous intensity group of STRRIDE I; (2) resistance training, in which subjects completed a regimen of three sessions per week during which nine resistance exercises were performed with eight to twelve repetitions at $70-85 \%$ of one repetition maximum weight; (3) low volume/vigorous intensity aerobic exercise plus resistance training, during which subjects completed the low volume/vigorous intensity aerobic training protocol in addition to the resistance training protocol; and (4) high volume/vigorous intensity aerobic training, identical to that of STRRIDE I.

For the statistical analyses in which the STRRIDE I and STRRIDE II datasets were combined, the groups were coded as follows: (1) STRRIDE I inactive controls; (2) STRRIDE I low volume/moderate intensity aerobic exercise; (3) STRRIDE I 
TABLE 1 | Characteristics of Subjects in STRRIDE I and STRRIDE || (\% (N) or mean \pm SD).

\begin{tabular}{|c|c|c|c|c|c|c|c|}
\hline & \multicolumn{2}{|c|}{ STRRIDE I } & \multicolumn{2}{|c|}{ STRRIDE II } & \multicolumn{2}{|l|}{ Total } & \multirow{2}{*}{$\begin{array}{c}\text { Gene Expression } \\
\text { Subset }\end{array}$} \\
\hline & $\begin{array}{l}\text { Failed to Complete } \\
\text { Study }\end{array}$ & $\begin{array}{l}\text { Completed } \\
\text { Study }\end{array}$ & $\begin{array}{c}\text { Failed to Complete } \\
\text { Study }\end{array}$ & $\begin{array}{l}\text { Completed } \\
\text { Study }\end{array}$ & $\begin{array}{l}\text { Failed to Complete } \\
\text { Study }\end{array}$ & $\begin{array}{l}\text { Completed } \\
\text { Study }\end{array}$ & \\
\hline & $28(67)$ & 72 (172) & $38(93)$ & 62 (153) & 33 (160) & 67 (325) & $n=60$ \\
\hline Age (y) & $51.3 \pm 5.8$ & $52.5 \pm 4.8$ & $46.5 \pm 11.8$ & $48.8 \pm 10.2$ & $48.5 \pm 10.0$ & $50.8 \pm 8.6$ & $51 \pm 1.2$ \\
\hline Gender $^{\star \star}$ - men & $40(27)$ & $55(94)$ & $35(33)$ & $44(68)$ & $37(60)$ & $50(162)$ & $50(30)$ \\
\hline Women & $60(40)$ & $45(78)$ & $65(60)$ & $56(85)$ & $63(100)$ & 50 (163) & $50(30)$ \\
\hline Race $^{\star \star}$ - white & 58 (39) & 81 (139) & $67(62)$ & $86(131)$ & $63(101)$ & 83 (270) & $90(54)$ \\
\hline Black & $42(28)$ & $19(33)$ & $33(31)$ & $14(22)$ & 37 (59) & (17) 55 & $10(6)$ \\
\hline $\begin{array}{l}\text { Body mass } \\
\text { Index (kg/m2) }\end{array}$ & $30.8 \pm 3.6$ & $29.9 \pm 2.9$ & $30.6 \pm 3.3$ & $30.5 \pm 3.3$ & $30.8 \pm 3.4$ & $30.2 \pm 3.1$ & $30.6 \pm 0.4$ \\
\hline $\begin{array}{l}\text { Minimum Waist } \\
\text { Circumference (cm) }\end{array}$ & $96.2 \pm 10.7$ & $95.4 \pm 9.8$ & $95.7 \pm 10.2$ & $96.4 \pm 9.6$ & $96.0 \pm 10.4$ & $95.9 \pm 9.7$ & $96.9 \pm 1.2$ \\
\hline $\begin{array}{l}\text { Pre-intervention peak } \mathrm{VO}_{2} \\
(\mathrm{~mL} / \mathrm{kg} / \mathrm{min})\end{array}$ & $26.4 \pm 6.1$ & $27.9 \pm 6.1$ & $26.6 \pm 8.8$ & $27.5 \pm 6.0$ & $26.5 \pm 6.4$ & $27.7 \pm 6.0$ & $27.6 \pm 0.7$ \\
\hline
\end{tabular}

${ }^{* *} p$-values for two sample t-test (completers vs. non-completers) significant in STRRIDE I, STRRIDE II, and total.

plus STRRIDE II low volume/vigorous intensity aerobic exercise groups; (4) STRRIDE I plus STRRIDE II high volume/vigorous intensity aerobic exercise groups; (5) STRRIDE II resistance training group; (6) STRRIDE II low volume/vigorous intensity aerobic exercise plus resistance exercise group. Demographics and physiologic characteristics of the groups are shown in Table 1.

\section{Biologic Measures}

All phenotypic measures were taken prior to initiation and at completion of the exercise intervention (Month 6 in STRRIDE I and Month 8 in STRRIDE II). Peak $\mathrm{VO}_{2}$ was measured using a graded treadmill exercise testing protocol with gas exchange analysis (Duscha et al., 2005). Exercise compliance was measured as a percentage of the assigned exercise minutes per week completed by the subject averaged over the intervention period. Non-completion occurred when a subject withdrew from further participation in the study for any of the following reasons: time constraints; injury or illness unrelated to the study; medical problems; family issues; or geographic relocation. No subjects were lost to follow- up.

\section{Genotyping}

DNA was isolated from whole blood using a commercial DNA isolation kit and a standard protocol (Qiagen, Inc, Valencia, CA). Acid ceramidase (ASAH1) SNPs were selected using the SNPSelector program in which a tagging algorithm prioritised SNPs for low linkage disequilibrium in the HapMap database, allelic frequencies, and regulatory potential (Xu et al., 2005). Six haplotype tagging SNPs in ASAH1 were identified: rs7844023, rs2898458, rs7508, rs3810, rs2427746, and rs1049874; genotypes were determined using the Taqman assay (Applied Biosystems, Foster City, CA). The Taqman genotyping reaction was then amplified using a GeneAmp PCR system $9700\left(95^{\circ} \mathrm{C}\right.$ for $10 \mathrm{~min}$, then $50 \mathrm{cycles}$ at $92^{\circ} \mathrm{C}$ for $15 \mathrm{~s}, 60^{\circ} \mathrm{C}$ for $\left.1 \mathrm{~min}\right)$. Fluorescence was detected using the $7900 \mathrm{HT}$ Taqman sequence detector (Applied
Biosystems). Two reference controls were included. All SNPs were successfully genotyped for $95 \%$ or more of the individuals in the study; rescreening of $2.4 \%$ of subjects gave $100 \%$ identical results. Error rate estimates for SNPs meeting the reference control benchmarks were $<0.2 \%$.

\section{Gene Expression Profiling}

We conducted gene expression analysis on a subset of 60 representative individuals randomly selected for further study from both data sets. The gene expression subgroup included 10 subjects (five men and five women) from each of the six exercise groups described above; paired baseline and posttraining samples were always processed in the same assay. Demographics for this cohort were not significantly different from those of the entire cohort (Table 1).

Total RNA was extracted using the standard Trizol (Invitrogen, Carlsbad, CA) method and 30 to $50 \mathrm{mg}$ of starting skeletal muscle. Two round amplification of total RNA was performed using a commercially available kit (Affymetrix, Santa Clara, CA). Thirty micrograms of biotinylated cRNA from each sample was hybridised to Affymetrix U133 Plus 2.0 microarrays. More detailed methods associated with microarray gene expression analysis can be found elsewhere (Hittel et al., 2005). Probe set expression levels were generated using the PLIER algorithm (typically 6 iterations) in Expression Console (Affymetrix) and imported directly into Partek Genomics Suite (Partek Inc., St. Louis, MO) for statistical processing.

\section{Statistical Analysis}

Haploview (27) was used to assess LD between SNPs using the combined STRRIDE I and STRRIDE II datasets. Genotype association with the non-completion study was analysed using a logistic regression model (SAS software, SAS Institute, Cary, NC). STRRIDE I and STRRIDE II datasets were analysed separately. As independent datasets, they provided an opportunity for validation of significance of individual SNPs in direction 
and magnitude of effect: STRRIDE II was considered the testing/exploratory set, and STRRIDE I the validation set. The outcome of non-completion of the exercise intervention was defined as a dichotomous variable with individuals who did not complete the intervention coded as 1 ; individuals who completed the program were coded as 0 . Genotypes were coded using an additive model with 0,1 , or 2 copies of one allele and using a dominant model for the presence vs. absence of the same allele. Race, gender, and exercise group were included in the regression model; these variables differed significantly in those subjects completing the study vs. non-completers (Table 1). Due to the very small numbers of individuals reporting Asian or Hispanic ethnicity, only subjects who were either black or white were included in the analysis. To examine the potential for confounding by race, the logistic regression analyses were also performed stratified by race. Odds ratios were estimated for non-completion vs. completion.

Multivariable linear regression models (SAS software, SAS Institute, Cary, NC) were used to model genotype association with peak oxygen consumption (peak $\mathrm{VO}_{2}$ ) and exercise compliance in completers. Genotypes were coded for additive and dominant models. As described above, all models included terms for gender, race, and exercise group. Given the consistency of the effects observed for the SNPs associated with noncompletion between STRRIDE I and STRRIDE II, the datasets were combined for analysis of change in peak $\mathrm{VO}_{2}$. Peak $\mathrm{VO}_{2}$ improvement models were tested with the complete dataset and with a dataset that excluded those subjects in the control and resistance training groups. Results are presented as mean $\pm \mathrm{SE}$.

Expression profile statistical analysis used Partek Genomics Suite (Version 6.4). Expression profiles were analysed to test differences in baseline gene expression between genotype groups. Following the gene expression value normalization, we used analysis of variance (ANOVA) with sex and race as covariates to examine genotype influences on mRNA expression also known as an eQTL analysis. $P<0.01$ were considered statistically significant in this analysis.

\section{RESULTS}

\section{Allele Frequencies}

For a complete gene analysis of the acid ceramidase gene, six ASAH1 SNPs were genotyped (rs7844023, rs2898458, rs7508, rs3810, rs2427746, rs1049874) in 239 subjects in STRRIDE I and 246 subjects in STRRIDE II. All allele frequencies were in Hardy Weinberg equilibrium (chi ${ }^{2}$ test, $P>0.05$ ) except for $\mathrm{rs} 1049874$ (Table 2). The rs1049874 SNP met all quality control benchmarks for genotyping as evaluated by an independent lab supervisor; it was therefore included in the analysis. Three SNPs, rs2898458, rs7508, and rs3810 were in pair-wise LD in whites $\left(r^{2}>0.60\right)$. Only SNPs rs2898458 and rs3810 were in strong LD in blacks $\left(r^{2}>0.70\right.$; Figure 1).

\section{Genetic Association With Non-completion: Exploratory Analysis-STRRIDE II}

Three ASAH1 SNPs, rs2898458, rs7508, and rs3810-in LDwere consistently associated with intervention non-completion in both additive and dominant models (Table 3). After controlling for group, gender, and race, each additional $\mathrm{T}$ allele at rs3810 doubled the odds of failure to complete; in the dominant model, subjects with either one or two copies of the T allele were 3.5 times more likely to fail to complete than those with the GG genotype (additive model $P=0.01$; dominant model $P=0.005$; Figure 2). Similarly, both rs2898458 allele $G$ and rs7508 allele $G$ significantly increased the odds of non-completion in both additive $(\mathrm{OR}=1.8,1.8 ; P=0.03,0.002$, respectively) and dominant $(\mathrm{OR}=2.5,2.6 ; P=0.02,0.02$, respectively) models.

\section{Genetic Association With Study Non-completion: Validation Analysis-STRRIDE I}

ASAH1 genotype associations were validated in the STRRIDE I dataset, in which the additive model was significantly associated with non-completion for rs3810 and rs2898458 (OR = 1.8, 1.8; $P=0.02,0.02)$. When comparing the direction and magnitude of association of each SNP, the two independent datasets showed excellent agreement, providing strong replication for association of these SNPs with failure to complete (Table 3, Figure 3). The race-stratified analyses supported the combined analysis with the white race group showing results consistent with the analysis of the full datasets for both STRRIDE I and STRRIDE II (supplemental tables).

\section{Genetic Association With Peak Oxygen Consumption in Intervention Completers}

To investigate potential mediators of the genetic association with failure-to-complete an exercise intervention, we studied the effects of the $A S A H 1$ variants on baseline peak $\mathrm{VO}_{2}$ and change in peak $\mathrm{VO}_{2}$ with exercise training; we hypothesised that poor exercise capacity or ability to respond to exercise training might explain why persons chose to not complete the protocol. Baseline peak $\mathrm{VO}_{2}$ was not significantly associated with genotype or study completion (data not shown). However, among completers, improvement in peak $\mathrm{VO}_{2}$ was correlated with genotype for two of the three ASAH1 SNPs previously associated with noncompletion. When compared to subjects homozygous for the $\mathrm{G}$ allele for SNP rs3810 and controlling for race, gender, group, and baseline peak $\mathrm{VO}_{2}$, each additional $\mathrm{T}$ allele decreased the improvement in peak oxygen consumption by $1.4(\mathrm{~mL} / \mathrm{kg} / \mathrm{min})$ $\left(p=0.0185, r^{2}=0.3689\right)$. Similarly, the G allele of SNP rs 2898458 showed a smaller improvement in peak $\mathrm{VO}_{2}(0.45 \mathrm{~mL} / \mathrm{kg} / \mathrm{min}$ decrease, $P=0.0734, r^{2}=0.362$ ).

One would not expect peak $\mathrm{VO}_{2}$ to change significantly in those subjects assigned to either the control or resistance training groups; we therefore tested the association of improvement in peak $\mathrm{VO}_{2}$ with genotype in the dataset excluding these two groups. Indeed, the two SNPs mentioned previously remained correlated with improvement in peak $\mathrm{VO}_{2}\left(\mathrm{rs} 3810 r^{2}=0.29\right.$, $P=0.036$; rs2898458 $r^{2}=0.29, P=0.0834$; Figure 4). In this model, rs7508 genotype was also associated with improvement in peak $\mathrm{VO}_{2}\left(p=0.0873, r^{2}=0.28\right)$. 
TABLE 2 | Genotype Frequency by Race.

\begin{tabular}{|c|c|c|c|c|c|}
\hline SNP & Genotype & \multicolumn{2}{|c|}{ STRRIDE I $(N=239)$} & \multicolumn{2}{|c|}{ STRRIDE II $(N=246)$} \\
\hline & CT & 46.5 & 46.6 & 53.2 & 40.4 \\
\hline & CT & 27.0 & 27.6 & 22.3 & 28.8 \\
\hline & T Allele frequency & 50.3 & 50.8 & 48.9 & 49.0 \\
\hline & $G G$ & 11.5 & 51.9 & 8.0 & 40.0 \\
\hline & G Allele frequency & 30.6 & 72.2 & 31.7 & 59.0 \\
\hline \multirow[t]{3}{*}{ RS7508 } & $\mathrm{AA}$ & 59.4 & 81.0 & 53.7 & 90.4 \\
\hline & $A G$ & 32.9 & 13.8 & 40.5 & 9.6 \\
\hline & $G G$ & 7.7 & 5.2 & 5.8 & 0.0 \\
\hline \multirow{2}{*}{ RS3810 } & $\pi$ & 12.9 & 53.6 & 8.5 & 43.1 \\
\hline & T Allele Frequency & 33.8 & 73.2 & 16.2 & 65.7 \\
\hline \multirow[t]{4}{*}{ RS2427746 } & AA & 35.5 & 63.0 & 27.5 & 70.6 \\
\hline & $A G$ & 41.4 & 29.6 & 49.7 & 27.4 \\
\hline & GG & 23.1 & 7.4 & 22.8 & 2.0 \\
\hline & G Allele Frequency & 43.8 & 22.2 & 47.6 & 15.6 \\
\hline \multirow[t]{4}{*}{ RS1049874 } & $\mathrm{CC}$ & 29.0 & 12.1 & 23.0 & 54.9 \\
\hline & CT & 36.7 & 31.0 & 51.3 & 37.2 \\
\hline & $\Pi$ & 34.3 & 56.9 & 25.7 & 7.8 \\
\hline & C Allele Frequency & 47.3 & 27.6 & 51.3 & 26.5 \\
\hline
\end{tabular}

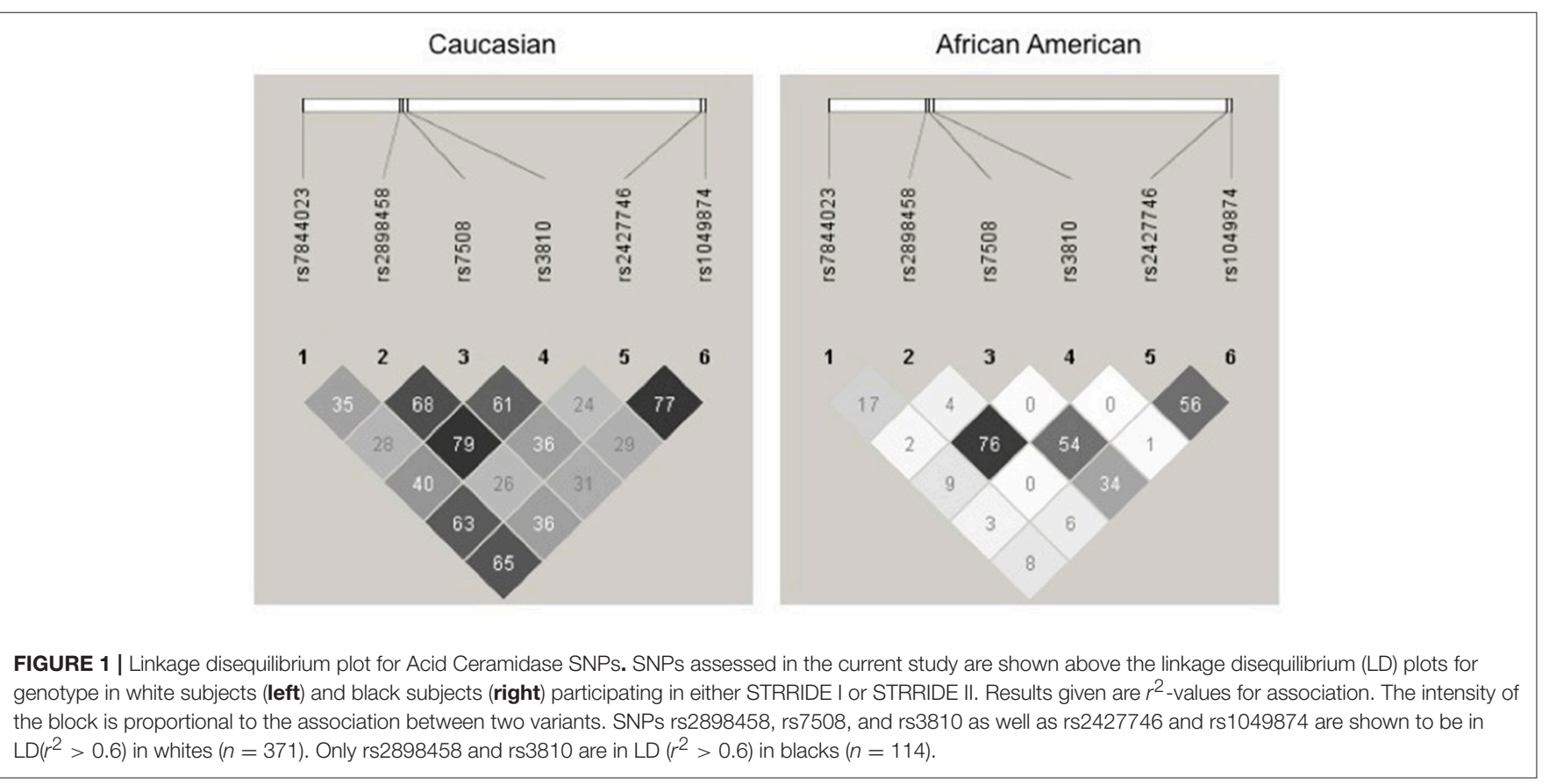


TABLE 3 | Genotype association with risk of failure to complete study, controlling for race, gender, and intervention group.

\begin{tabular}{|c|c|c|c|c|c|}
\hline & & \multicolumn{2}{|c|}{ Additive Model } & \multicolumn{2}{|c|}{ Dominant Model } \\
\hline & & Odds Ratio & $P$-value & Odds Ratio & $P$-value \\
\hline \multirow[t]{2}{*}{ rs7844023 $(\mathrm{C} / \mathbf{T})^{\star}$} & STRRIDE I & 0.88 & 0.562 & 0.88 & 0.300 \\
\hline & STRRIDE II & 0.86 & 0.513 & 0.82 & 0.596 \\
\hline \multirow[t]{2}{*}{ rs2898458 (A/G) } & STRRIDE I & $1.80^{\star}$ & $0.022^{*}$ & 1.80 & 0.384 \\
\hline & STRRIDE II & $1.79^{\star}$ & $0.026^{\star}$ & $2.52^{\star}$ & $0.018^{*}$ \\
\hline \multirow[t]{2}{*}{ rs7508 (A/G) ${ }^{\star}$} & STRRIDE I & 1.17 & 0.546 & 1.17 & 0.499 \\
\hline & STRRIDE ॥ & $1.78^{\star}$ & $0.034^{*}$ & $2.55^{\star}$ & $0.015^{\star}$ \\
\hline \multirow[t]{2}{*}{ rs3810 $(\mathrm{G} / \mathbf{T})^{\star}$} & STRRIDE I & $1.79^{\star}$ & $0.016^{\star}$ & 1.79 & 0.687 \\
\hline & STRRIDE II & $2.02^{*}$ & $0.012^{*}$ & $3.49^{*}$ & $0.005^{\star}$ \\
\hline \multirow[t]{2}{*}{ rs2427746 (A/G) } & STRRIDE I & 0.95 & 0.838 & 0.95 & 0.455 \\
\hline & STRRIDE ॥ & 1.17 & 0.543 & 1.15 & 0.711 \\
\hline \multirow[t]{2}{*}{ rs1049874 (T/C)* } & STRRIDE I & 1.11 & 0.635 & 1.11 & 0.92 \\
\hline & STRRIDE II & 1.18 & 0.628 & 1.21 & 0.628 \\
\hline
\end{tabular}

All models controlled for race, gender and intervention group. Significant effects are marked with asterisk (*) and indicated in bold.

\section{Genetic Effects on mRNA Expression of ASAH1}

We generated gene expression profiles using a subset of 60 individuals from both STRRIDE I and STRRIDE II. As shown in Table 1, demographic data for age, height, weight, and BMI were not different between the total cohort and the gene expressionprofiled sub-cohort. Peak $\mathrm{VO}_{2}$ at baseline was lower in the gene expression subgroup than the total population; however, it was not different between the subgroup and all subjects that completed the study. There are five probe sets for ASAH1 on the U133 Plus 2.0 microarray, of which only two (213702_x_at and 210979_at) target the full length ASAH1 transcript and are highly expressed in skeletal muscle. Expression of these two probe sets was significantly different between rs3810 genotypes, when tested with either an additive $(p=0.006$ and $p=0.007$ for probe sets 213702_x_at and 210979_at, respectively) or a dominant model ( $p=0.00007$ and $p=0.0001$ for probe sets 213702_x_at and 210979_at, respectively). For rs3810, the TT group demonstrated between a 1.3 and 1.4 times reduction in ASAH1 mRNA expression when compared with the GG group (Figure 5). Linkage disequilibrium between rs3810 and rs2898458 was nearly complete in the gene expression subgroup. Therefore, similar results were found for the relation between skeletal muscle $A S A H 1$ expression and rs2898458 genotype (data not shown). Linkage disequilibrium between rs3810 and rs7508 was somewhat lower at 78\%, with only one GG subject (also homozygous for the minor allele for both other SNPs); however, the gene expression results for rs7508 were similar to those for rs3810 (not shown).

\section{DISCUSSION}

In the present study, we provide evidence suggesting that genetic variation within the acid ceramidase gene is associated with an individual's ability or willingness to persist in a newly prescribed exercise program. Three of six ASAH1 SNPs (rs2898458, rs3810,

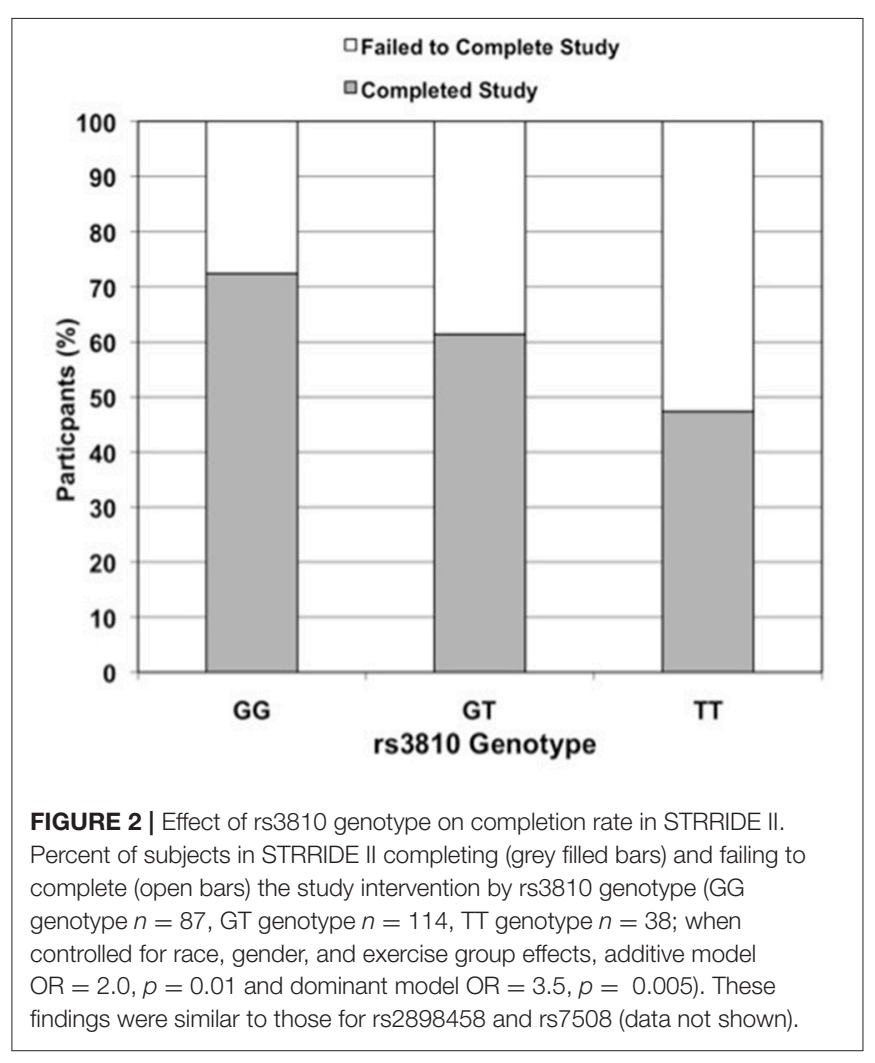

and rs7508; all in LD) were associated with significantly increased risk of non-completion of an exercise intervention in two exercise training interventions - STRRIDE I and STRRIDE II. These SNPs were also associated with change in peak $\mathrm{VO}_{2}$ among the completers; there also were differences in ASAH1 gene expression such that genetic correspondence with gene expression was exactly consistent with less improvement in $\mathrm{VO}_{2}$ with training and greater non-completion rates. Thus, these findings support 


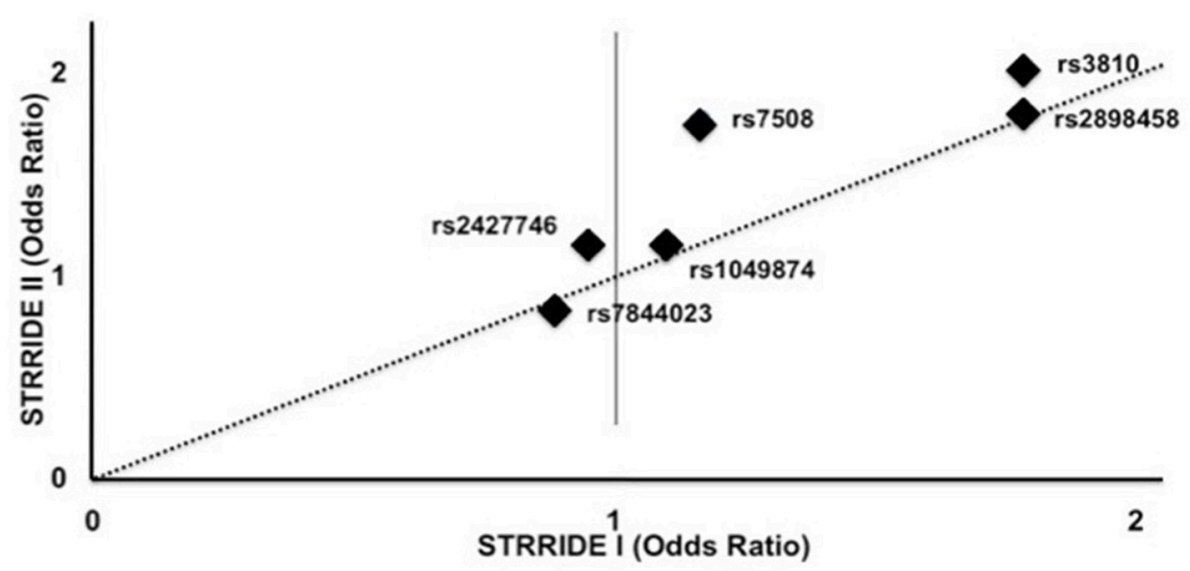

FIGURE 3 | Genotype effect on odds of failure to complete study by SNP in STRRIDE II vs. STRRIDE I. Additive model odds ratios for risk of study non-completion for each ASAH SNP for STRRIDE I ( $x$ axis) and STRRIDE II (y axis). Two SNPs significantly increased the odds of failure to complete both STRRIDE II and STRRIDE I: rs2898458 and rs3810. SNP rs7508 significantly increased the odds of study non-completion in STRRIDE II.

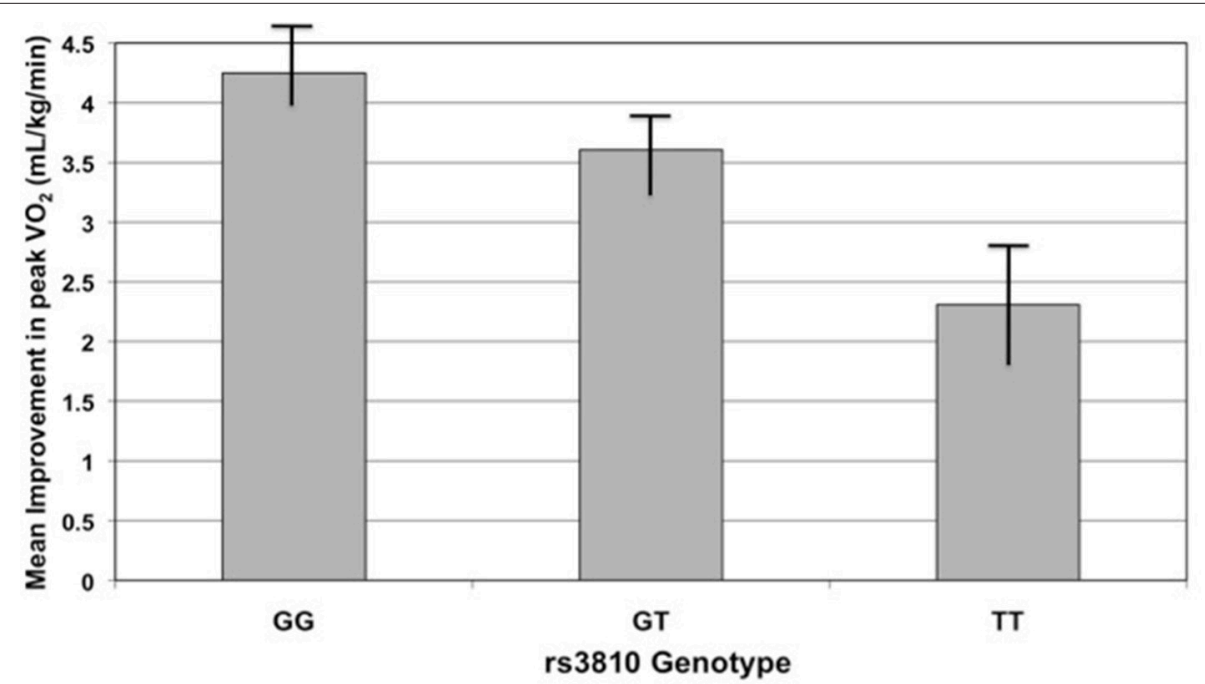

FIGURE 4 | Improvement in peak oxygen consumption (peak $\mathrm{VO}_{2}$ ) with exercise training by $\mathrm{rs} 3810$ genotype. Improvement in peak VO $\mathrm{I}_{2}$ ( $\mathrm{mL} / \mathrm{kg} / \mathrm{min}$ ), measured as the difference in peak $\mathrm{VO}_{2}$ before and after an exercise intervention, compared to rs3810 genotype. STRRIDE I and STRRIDE II datasets were combined for the analysis (GG genotype $n=168$, GT genotype $n=207$, TT genotype $n=90$ ). The rs3810 genotype significantly predicted change in peak VO 2 ( $p=0.0185)$, despite the fact that some groups differed in the intensity of the training stimulus.

plausible biological links between genetic variation in $A S A H 1$ and exercise behaviour.

Ceramide is produced either via hydrolysis of membrane sphingomyelin; or de novo from long- chain saturated fatty acids. In non-adipocytes, increased fatty acid deposition with subsequent conversion to ceramide has been proposed as a mechanism of lipotoxicity in type 2 diabetes, heart failure, and atherosclerosis (Zhou et al., 2000). Accumulation of ceramide initiates pro-apoptotic pathways converging on mitochondrial function-the major source of pro-apoptotic molecules (e.g., BCL1). (Birbes et al., 2002). Increased intracellular ceramide leads to the apoptosis of pancreatic beta cells and cardiac myocytes in obese rats leading to decreased function of both cell types (Unger et al., 1999; Zhou et al., 2000). Ceramide also directly inhibits both complex I and complex III of the mitochondrial respiratory chain in rat heart muscle in vitro (Gudz et al., 1997; Di Paola et al., 2000).

Acid ceramidase, ubiquitously expressed in somatic cells, plays a crucial role in the maintenance of cellular ceramide concentrations (Park and Schuchman, 2006). Acid ceramidase metabolizes ceramide into sphingosine and free fatty acids. Overexpression of the enzyme prevents the inhibitory effects of accumulated saturated fatty acids on insulin signalling(Chavez et al., 2005) while abnormally high expression of the enzyme has been reported in several human cancers (Park and Schuchman, 2006). Ceramide content is increased in skeletal muscle of 


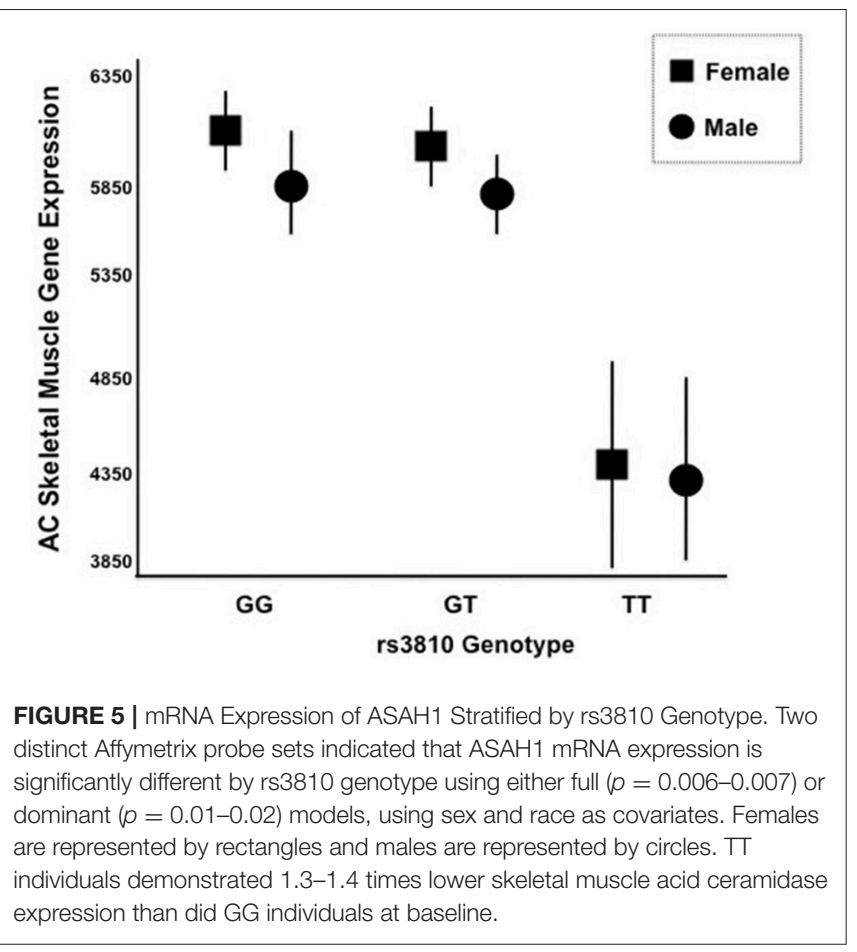

obese, insulin resistant humans (Adams et al., 2004) and endurance exercise reduces the content of ceramide in skeletal muscle in obese subjects with a concomitant improvement in insulin sensitivity (Bruce et al., 2006). Nonetheless, ceramide relationships to insulin sensitivity and changes with exercise are complex and incompletely understood: effects appear dependent on ceramide species, exercise duration and intensity, and underlying insulin sensitivity (Dobrzyn and Gorski, 2002a; Helge et al., 2004; Baranowski et al., 2008; BłachnioZabielska et al., 2008; Bergman et al., 2016). Further, in addition to acid ceramidase, ceramide regulation can occur via degradation by neutral and alkaline ceramidases or conversion to sphingomyelin.

It is important to recognize that we have not necessarily found functional acid ceramidase variants; rather, we only have identified and replicated associations implicating the acid ceramidase gene in the biological response. Nonetheless, in light of recognised acid ceramidase functions, it is intriguing that acid ceramidase polymorphisms were associated with reduced completion rates for and poorer peak oxygen consumption (peak $\mathrm{VO}_{2}$ ) responses to exercise training. All three significant acid ceramidase SNPs were associated with differential improvements in peak $\mathrm{VO}_{2}$ with exercise. While only one of the SNPs was significant in this association, one should note that post-intervention peak $\mathrm{VO}_{2}$ measurements were available for exercise completers only. Were it feasible to include both exercise completers and non-completers in the post-intervention peak $\mathrm{VO}_{2}$ analysis, the correlation between acid ceramidase minor allelic genotype and decreased improvement in oxygen consumption may have been stronger for all three genotypes.
There were significant differences in racial groups for non-completion rates, as well as large differences in allele frequencies for rs3810 and rs2898458; this raised the issue of the potential for allele frequency differences to confound the strong association observed for these variants. To evaluate the stability of the associations observed in the overall group, we performed race-stratified analyses for each study. In both whites and blacks, rs3810 demonstrated association with noncompletion; the odds ratios for the same alleles in both races and both studies were very consistent. The non-significant $p$ values in the smaller black subgroup reflected differences in allele frequency and sample size. Although detailed evaluation of genetic variation across $A S A H 1$ will be required to identify functional SNPs, comparing the results for blacks and whites in light of the expected differences in linkage disequilibrium patterns provided specific support for rs3810 as the SNP of interest in ASAH1.

We routinely queried participants for their reasons when withdrawing from the study prior to completion. The reasons varied considerably: among others were time constraints; family issues; "a changed mind"; or unrelated medical problems. Clearly, many issues can affect compliance with behavioural interventions-including exercise-not explainable by genetics alone. However, the association between study non-completion and acid ceramidase genotypes remained significant despite the "noise" created by the subjects' social environment and personality traits; this implies that the biological relation might be even stronger than our findings indicate. Furthermore, the association of the phenotypic measure of peak $\mathrm{VO}_{2}$ improvement with exercise compliance and acid ceramidase genotype points to a physiologic mechanism for exercise intolerance. Perhaps the genetically associated unresponsiveness to exercise training may consciously or subconsciously play into the willingness of individuals to continue to participate in an exercise program; this may be exacerbated by personality factors, life stress, or other environmental influences.

Personality characteristics may play into the interplay between genetics and exercise behaviour (Herring et al., 2014). Elements of the Big Five Personality Factors are associated with other lifestyle elements-dietary habits and smoking, among othersand also are associated with aerobic capacity (Terracciano et al., 2013) muscle strength (Tolea et al., 2012) and adherence to post-surgery rehabilitation (Hilliard et al., 2014). It would be important-and perhaps therapeutically useful-to know whether the half of individuals with the "drop-out genotype" that persisted with the intervention have a different personality profile than those that fail to complete the intervention. If genetic effects on exercise behaviour are mediated-at least in part-through personality factors; and if personality factors explain the variation in exercise adherence behaviour among those that are at genetic risk of poor adherence; then one might want to test whether personality factors can inform personalised strategies and messaging to increase adherence for those whose health would most benefit from increases in regular exercise. 
To our knowledge this is the first report of the effects of acid ceramidase polymorphisms on exercise behaviour in overweight to mildly obese, insulin resistant subjects. Our findings should be validated in subsequent studies. Given resource constraints and sample availability, we were unable to quantify acid ceramidase enzyme activity, per se, within our subjects. We also did not measure whether acid ceramidase gene variants differentially affected skeletal muscle enzyme or ceramide content in these subjects-either prior to or in response to an exercise intervention. Future studies will be necessary to further elucidate the relation between common gene variants of acid ceramidase with skeletal muscle enzyme activity, ceramide content, and physical performance.

In conclusion, these data suggest that genetic variation within the acid ceramidase gene significantly affects exercise tolerance and completion of an exercise program. Acid ceramidase regulates ceramide content within skeletal and cardiac muscle; these effects are likely to be involved in muscle adaptations to exercise training. Due to the proven health benefits of regular exercise, characterization of individual exercise potential conferred by genetic variation prior to initiation of an intervention may be helpful in maximizing the adherence to exercise and therefore the health benefits accrued therefrom.

\section{REFERENCES}

Adams, J. M. II., Pratipanawatr, T., Berria, R., Wang, E., DeFronzo, R. A., Sullards, M. C., et al. (2004). Ceramide content is increased in skeletal muscle from obese insulin-resistant humans. Diabetes 53, 25-31. doi: 10.2337/diabetes.53.1.25

Baranowski, M., Zabielski, P., Blachnio, A., and Gorski, J. (2008). Effect of exercise duration on ceramide metabolism in the rat heart. Acta Physiol. 192, 519-529. doi: 10.1111/j.1748-1716.2007.01755.x

Bergman, B. C., Brozinick, J. T., Strauss, A., Bacon, S., Kerege, A., Bui, H. H., et al. (2016). Muscle sphingolipids during rest and exercise: a C18:0 signature for insulin resistance in humans. Diabetologia 59, 785-798. doi: $10.1007 / \mathrm{s} 00125-015-3850-y$

Birbes, H., El Bawab, S., Obeid, L. M., and Hannun, Y. A. (2002). Mitochondria and ceramide: intertwined roles in regulation of apoptosis. Adv. Enzyme Regul. 42, 113-129. doi: 10.1016/S0065-2571(01)00026-7

Błachnio-Zabielska, A., Baranowski, M., Zabielski, P., and Górski, J. (2008). Effect of exercise duration on the key pathways of ceramide metabolism in rat skeletal muscles. J. Cell. Biochem. 105, 776-784. doi: 10.1002/jcb.21877

Błachnio-Zabielska, A., Zabielski, P., Baranowski, M., and Gorski, J. (2011). Aerobic training in rats increases skeletal muscle sphingomyelinase and serine palmitoyltransferase activity, while decreasing ceramidase activity. Lipids. 46,229-238. doi: 10.1007/s11745-010-3515-Z

Bouchard, C., Blair, S. N., Church, T. S., Earnest, C. P., Hagberg, J. M., Häkkinen, K., et al. (2012). Adverse respoçnse to regular exercise: is it a rare or common occurrence? PLoS ONE 7:e37887. doi: 10.1371/journal.pone.0037887

Bouchard, C., Sarzynski, M. A., Rice, T. K., Kraus, W. E., Church, T. S., Sung, Y. J., et al. (2011). Genomic predictors of the maximal O2 uptake response to standardised exercise training programs. J. Appl. Physiol. 110, 1160-1170. doi: 10.1152/japplphysiol.00973.2010

Bruce, C. R., Thrush, A. B., Mertz, V. A., Bezaire, V., Chabowski, A., Heigenhauser, G. J., et al. (2006). Endurance training in obese humans improves glucose tolerance and mitochondrial fatty acid oxidation and alters muscle lipid content. Am. J. Physiol. Endocrinol. Metab. 291, E99-E107. doi: 10.1152/ajpendo.00587.2005

Chavez, J. A., Holland, W. L., Bär, J., Sandhoff, K., and Summers, S. A. (2005). Acid ceramidase overexpression prevents the inhibitory effects of

\section{ETHICS STATEMENT}

Informed consent was obtained under protocols approved by the Investigational Review Boards of Duke University and East Carolina University.

\section{AUTHOR CONTRIBUTIONS}

WK and MD conceived the science. WK and EPH financial support for the research. WK and LL wrote manuscript. WK, CS, $\mathrm{JH}, \mathrm{LL}, \mathrm{ICS}$, and MH conducted experiment. LL, ERH, and MH performed analysis. WK, KH, and ICS edited manuscript.

\section{ACKNOWLEDGMENTS}

We thank the entire STRRIDE team at Duke and ECU for assistance with conduct of the study and for the participants for their substantial contributions. We thank our wonderful study participants. We acknowledge the support of the following funding agencies for support of portions of this project or personnel: NIH/NHLBI 1R01HL57354 and 2R01HL57354, NIH/NIA AG028930; NIH/NIAMS F32 AR052596 (MH); NIH/NCRR UL1RR024128 (LL) and, NIHNIAMSK.23A. R.054904 (KH).

saturated fatty acids on insulin signaling. J. Biol. Chem. 280, 20148-20153. doi: $10.1074 /$ jbc.M412769200

DHHS (2008). Physical Activity Guidelines Advisory Committee Report. Washington, DC: DHHS.

Di Paola, M., Cocco, T., and Lorusso, M. (2000). Ceramide interaction with the respiratory chain of heart mitochondria. Biochemistry 39, 6660-6668. doi: 10.1021/bi9924415

Dobrzyn, A., and Gorski, J. (2002a). Effect of acute exercise on the content of free sphinganine and sphingosine in different skeletal muscle types of the rat. Horm. Metab. Res. 34, 523-529. doi: 10.1055/s-2002-34793

Dobrzyn, A., and Gorski, J. (2002b). Ceramides and sphingomyelins in skeletal muscles of the rat: content and composition. Effect of prolonged exercise. Am. J. Physiol. Endocrinol. Metab. 282, E277-E285. doi: 10.1152/ajpendo.0015 1.2001

Duscha, B. D., Slentz, C. A., Johnson, J. L., Houmard, J. A., Bensimhon, D. R., Knetzger, K. J., et al (2005). Effects of exercise training amount and intensity on peak oxygen consumption in middle-age men and women at risk for cardiovascular disease. Chest.128, 2788-2793. doi: 10.1378/chest.128.4.2788

Gudz, T. I., Tserng, K. Y., and Hoppel, C. L. (1997). Direct inhibition of mitochondrial respiratory chain complex III by cell-permeable ceramide. $J$. Biol. Chem. 272, 24154-24158. doi: 10.1074/jbc.272.39.24154

Hannun, Y. A., and Obeid, L. M. (2002). The Ceramide-centric universe of lipidmediated cell regulation: stress encounters of the lipid kind. J. Biol. Chem. 277, 25847-25850. doi: 10.1074/jbc.R200008200

Helge, J. W., Dobrzyn, A., Saltin, B., and Gorski, J. (2004). Exercise and training effects on ceramide metabolism in human skeletal muscle. Exp. Physiol. 89, 119-127. doi: 10.1113/expphysiol.2003.002605

Herring, M. P., Sailors, M. H., and Bray, M. S. (2014). Genetic factors in exercise adoption, adherence and obesity. Obesity Rev. 15, 29-39. doi: 10.1111/obr.12089

Hilliard, R. C., Brewer, B. W., Cornelius, A. E., and Van Raalte, J. L. (2014). Big five personality characteristics and adherence to clinic-based rehabilitation activities after acl surgery: a prospective analysis. Open Rehabil. J. 7, 1-5. doi: $10.2174 / 1874943701407010001$

Hittel, D. S., Kraus, W. E., Tanner, C. J., Houmard, J. A., and Hoffman, E. P. (2005). Exercise training increases electron and substrate shuttling proteins in muscle 
of overweight men and women with the metabolic syndrome. J. Appl. Physiol. 98, 168-179. doi: 10.1152/japplphysiol.00331.2004

Huffman, K. M., Koves, T. R., Hubal, M. J., Abouassi, H., Beri, N., Bateman, L. A., et al. (2014). Metabolite signatures of exercise training in human skeletal muscle relate to mitochondrial remodelling and cardiometabolic fitness. Diabetologia 57, 2282-2295. doi: 10.1007/s00125-014-3343-4

Kraus, W. E., Torgan, C. E., Duscha, B. D., Norris, J., Brown, S. A., Cobb, F. R., et al. (2001). Studies of a targeted risk reduction intervention through defined exercise (STRRIDE). Med. Sci. Sports Exerc. 33, 1774-1784. doi: 10.1097/00005768-200110000-00025

Mao, C., and Obeid, L. M. (2008). Ceramidases: regulators of cellular responses mediated by ceramide, sphingosine, and sphingosine-1-phosphate. Biochim. Biophys. Acta 1781, 424-434. doi: 10.1016/j.bbalip.2008.06.002

Park, J. H., and Schuchman, E. H. (2006). Acid ceramidase and human disease. Biochim. Biophys. Acta 1758, 2133-2138. doi: 10.1016/j.bbamem.2006.08.019

Summers, S. A. (2006). Ceramides in insulin resistance and lipotoxicity. Prog. Lipid Res. 45, 42-72. doi: 10.1016/j.plipres.2005.11.002

Terracciano, A., Schrack, J. A., Sutin, A. R., Chan, W., Simonsick, E. M., and Ferrucci, L. (2013). Personality, metabolic rate and aerobic capacity. PLoS ONE 2013:e54746. doi: 10.1371/journal.pone.0054746

Tolea, M. I., Terracciano, A., Simonsick, E. M., Metter, E. J., Costa, P. T. Jr., and Ferrucci, L. (2012). Associations between personality traits, physical activity level, and muscle strength. J. Res. Pers. 46, 264-270. doi: $10.1016 /$ j.jrp.2012.02.002
Unger, R. H., Zhou, Y. T., and Orci, L. (1999). Regulation of fatty acid homeostasis in cells: novel role of leptin. Proc. Natl. Acad. Sci. U.S.A. 96, 2327-2332. doi: 10.1073/pnas.96.5.2327

Xu, H., Gregory, S. G., Hauser, E. R., Stenger, J. E., Pericak-Vance, M. A., Vance, J. M., et al. (2005). SNPselector: a web tool for selecting SNPs for genetic association studies. Bioinformatics 21, 4181-4186. doi: 10.1093/bioinformatics/bti682

Zhou, Y. T., Grayburn, P., Karim, A., Shimabukuro, M., Higa, M., Baetens, D., et al. (2000). Lipotoxic heart disease in obese rats: implications for human obesity. Proc. Natl. Acad. Sci. U.S.A. 97, 1784-1789. doi: 10.1073/pnas.97. 4.1784

Conflict of Interest Statement: The authors declare that the research was conducted in the absence of any commercial or financial relationships that could be construed as a potential conflict of interest.

Copyright (c) 2018 Lewis, Huffman, Smith, Donahue, Slentz, Houmard, Hubal, Hoffman, Hauser, Siegler and Kraus. This is an open-access article distributed under the terms of the Creative Commons Attribution License (CC BY). The use, distribution or reproduction in other forums is permitted, provided the original author(s) and the copyright owner(s) are credited and that the original publication in this journal is cited, in accordance with accepted academic practice. No use, distribution or reproduction is permitted which does not comply with these terms. 